\title{
Erratum
}

\section{REM near-IR and optical multiband observations of PKS 2155-304 in 2005}

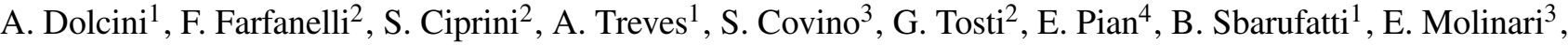 \\ G. Chincarini ${ }^{3,5}$, F. M. Zerbi ${ }^{3}$, G. Malaspina ${ }^{3}$, P. Conconi ${ }^{3}$, L. Nicastro ${ }^{6}$, E. Palazzi $^{6}$, V. Testa ${ }^{7}$, F. Vitali ${ }^{7}$, \\ L. A. Antonelli ${ }^{7}$, J. Danziger ${ }^{4}$, G. Tagliaferri ${ }^{3}$, E. Meurs ${ }^{8}$, S. Vergani ${ }^{8}$, A. Fernandez-Soto ${ }^{9}$, E. Distefano ${ }^{10}$, \\ G. Cutispoto ${ }^{10}$, and F. D'Alessio ${ }^{7}$ \\ 1 Università degli Studi dell'Insubria, Dipartimento di Fisica e Matematica, via Valleggio 11, 22100 Como, Italy \\ e-mail: alberto.dolcini@gmail.com \\ 2 Dipartimento di Fisica e Osservatorio Astronomico, Università di Perugia, via. A. Pascoli, 06123 Perugia, Italy \\ 3 INAF - Osservatorio Astronomico di Brera, via E. Bianchi 46, 23807 Merate (LC), Italy \\ 4 INAF - Osservatorio Astronomico di Trieste, via G. B. Tiepolo 11, 34143 Trieste, Italy \\ 5 Università degli Studi di Milano-Bicocca, Dipartimento di Fisica, Piazza delle Scienze, 3, 20126 Milan, Italy \\ INAF/IASF Bologna, via Gobetti 101, 40129 Bologna, Italy \\ 7 INAF - Osservatorio Astronomico di Roma, via Frascati 33, 00040 Monteporzio Catone, Italy \\ 8 Dunsink Observatory, Castleknock, Dublin 15, Ireland \\ 9 Observatori Astronomic, Universitat de Valencia, Aptdo. Correos 22085, Valencia 46071, Spain \\ 10 INAF - Osservatorio Astrofisico di Catania, via S. Sofia 78, 95123 Catania, Italy
}

A\&A 469, 503-510 (2007), DOI: 10.1051/0004-6361:20066477

Key words. galaxies: active - BL Lacertae objects: individual: PKS 2155-30 - errata, addenda

We have found an error in our VRI photometry of PKS 2155-30 due to incorrect transformation of data files. This has unfortunately propagated in various tables and figures.

Here we give the corrected version. The detailed photometry which was reported in electronic form at the CDS via anonymous ftp to cdsarc.u-strasbg.fr (130.79.128.5) or via http://cdsweb.u-strasbg.fr/cgi-bin/qcat?J/A+A/469/503 has also been corrected. All the conclusions of the paper remain unchanged. 
Table 3. Average intensities for all epochs and all filters. All data are in mJy units.

\begin{tabular}{rrrrr}
\hline \hline Filter & $H$ & $I$ & $R$ & $V$ \\
\hline Average & $114.9 \pm 3.3$ & $34.45 \pm 6.5$ & $30.89 \pm 5.13$ & $30.70 \pm 5.05$ \\
Max value & 156.5 & 46.4 & 38.3 & 37.4 \\
Min value & 36.5 & 19.1 & 16.2 & 16.2 \\
Average ep.1 & $39.3 \pm 1.4$ & $21.4 \pm 1.5$ & $18.7 \pm 1.3$ & $18.1 \pm 0.7$ \\
Average ep.2 & $65.9 \pm 5.2$ & $28.4 \pm 3.3$ & $27.2 \pm 2.5$ & $20.3 \pm 3.4$ \\
Average ep.3 & $122.9 \pm 6.1$ & $38.8 \pm 1.9$ & $34.1 \pm 1.5$ & $33.5 \pm 1.7$ \\
\hline
\end{tabular}

Table 4. Average intensities and 1-sigma values for all filters for all five nights with more intensive observations in November 2005 (all values are in mJy units).

\begin{tabular}{rrrrrr}
\hline \hline Night & $4 / 11$ & $8 / 11$ & $18 / 11$ & $19 / 11$ & $20 / 11$ \\
\hline Average $H$ & $119.3 \pm 1.7$ & $119.3 \pm 3.0$ & $120.4 \pm 2.1$ & $124.5 \pm 2.8$ & $130.8 \pm 3.0$ \\
Average $I$ & $39.1 \pm 0.6$ & $36.4 \pm 0.5$ & $38.7 \pm 0.6$ & $38.7 \pm 0.6$ & $38.3 \pm 0.7$ \\
Average $R$ & $38.5 \pm 0.8$ & $36.40 \pm .8$ & $37.3 \pm 0.5$ & $38.0 \pm 1.6$ & $37.1 \pm 0.5$ \\
Average $V$ & $33.2 \pm 1.1$ & $33.0 \pm 1.1$ & $33.4 \pm 1.1$ & $33.5 \pm 1.1$ & $34.7 \pm 0.1$ \\
\hline
\end{tabular}

Table 5. Spectral index values and $V$ values for all spectra plotted in Fig. 11.

\begin{tabular}{ccc}
\hline \hline Data set & $\alpha$ & $V(\mathrm{mJy})$ \\
\hline This work (13/5/2005) & $0.77 \pm 0.16$ & $16.485 \pm 0.263$ \\
This work (19/9/2005) & $0.88 \pm 0.05$ & $24.370 \pm 0.238$ \\
This work (4/11/2005) & $1.32 \pm 0.24$ & $35.278 \pm 0.498$ \\
Bertone at al. (2000) & $0.42 \pm 0.26$ & $26.20 \pm 0.58$ \\
Pesce et al. (1997) & $0.62 \pm 0.30$ & $24.50 \pm 0.67$ \\
Zhang \& Xie (1996) & $0.62 \pm 0.16$ & $22.90 \pm 0.63$ \\
Bersanelli et al. (1992) & $0.61 \pm 0.38$ & $51.88 \pm 1.56(\mathrm{~J}$ band $)$ \\
Treves et al. (1989) (1/12/1983) & $0.51 \pm 0.31$ & $19.80 \pm 0.36$ \\
Treves et al. (1989) (11/11/1984) & $0.51 \pm 0.41$ & $26.20 \pm 0.48$ \\
Miller \& McAlister (1983) & $0.62 \pm 0.56$ & 17.8 \\
\hline
\end{tabular}
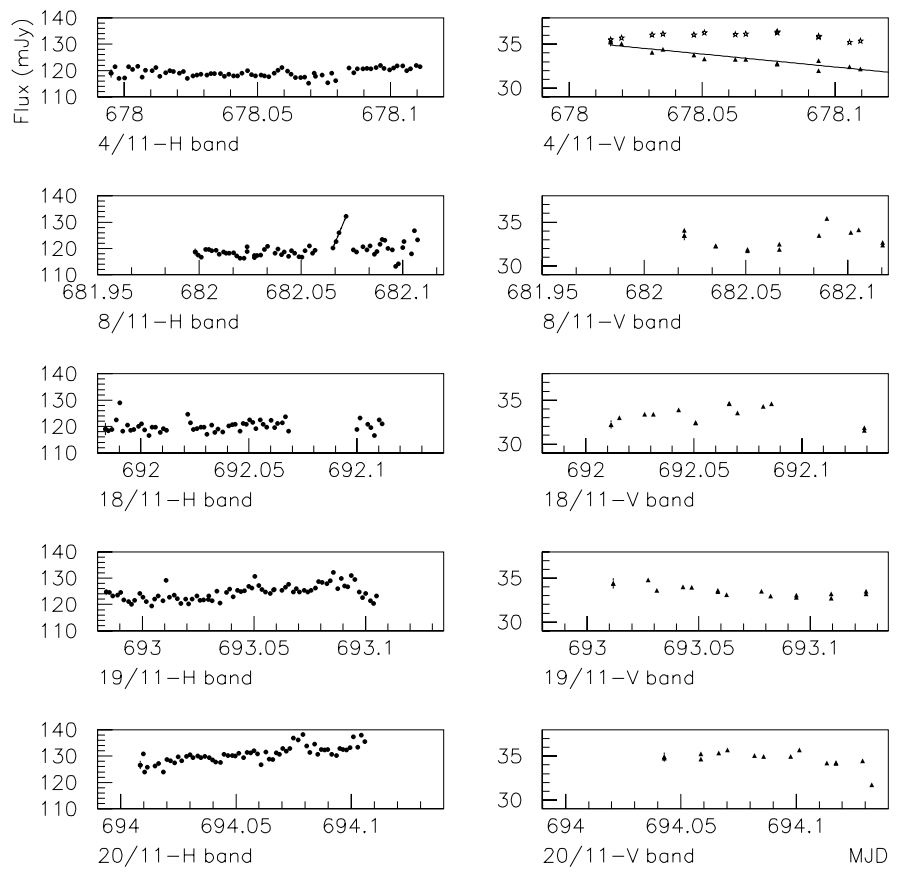

$19 / 11-V$ band

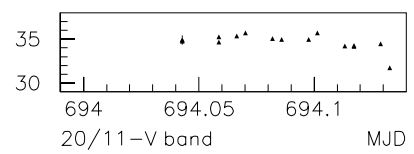

Fig. 4. Light curves in the $H$ and $V$ filters for five nigths in November 2005, when the observations were more intensive. Dates of observations are reported in each box. The solid line in $V$ band -4 Nov. box results from a linear regression analysis. The solid line in $H$ band 8 Nov. box connects the four points of the flare-like structure. In each box it is given a typical error bar. In $V$ band -4 Nov. box the light curve of one comparison star is also plotted, with a fixed enhancement of $9 \mathrm{mJy}$.

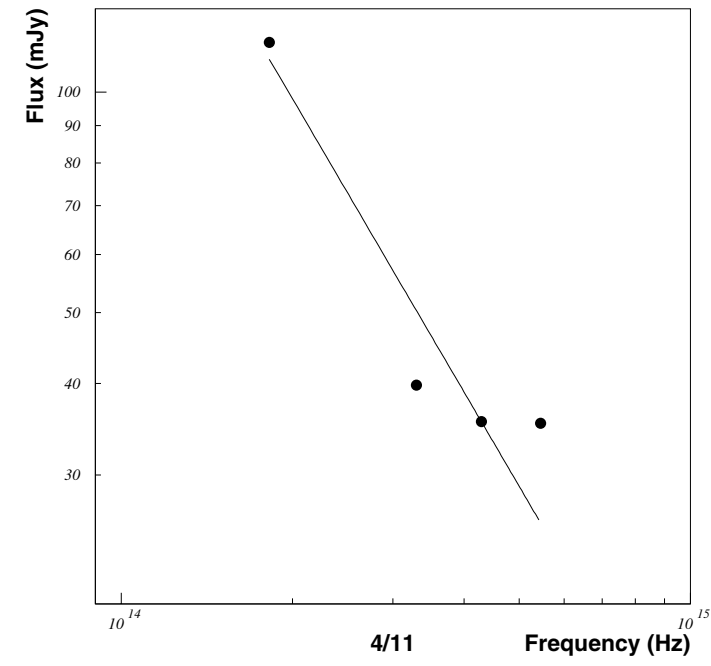

Fig. 10. 4 November 2005 spectrum - epoch 3. The spectral fit with a single power law yields a spectral index $\alpha=1.32 \pm 0.25$. Error bars are comparable with symbols size. 


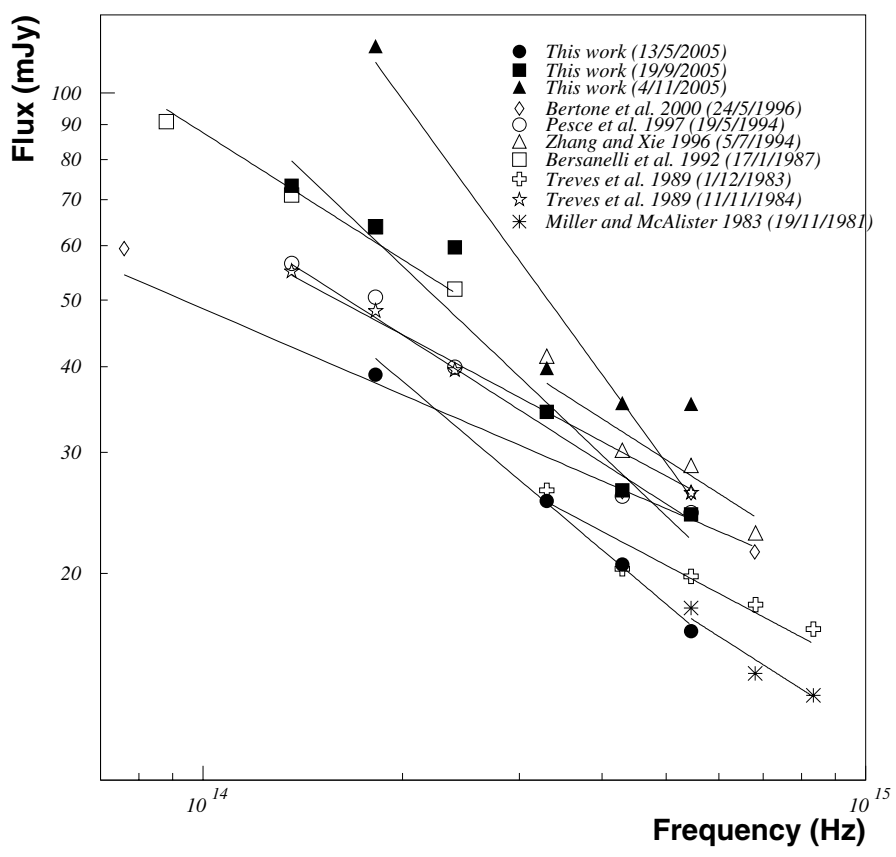

Fig. 11. Different spectra of PKS 2155-304 from observations at other epochs reported in the literature. Symbols correspond to following works: filled circles: this work (13/5/2005 data), filled squares: this work (19/9/2005 data), filled up triangles: this work (4/11/2005 data), open diamonds: Bertone et al. (2000; 24/5/1996 data), open circles: Pesce et al. (1997; 19/5/1994 data, the Hamuy \& Maza (1989) calibration is used), open up triangles: Zhang \& Xie (1996; 5/7/1994 data), open squares: Bersanelli et al. (1992; 17/1/1987 data), open crosses: Treves et al. (1989; 1/12/1983 data), open stars: Treves et al. (1989; 11/11/1984 data), asterisks: Miller \& McAlister (1983; 19/11/1981 data). Spectral index values and $V$ magnitudes for all data sets are reported in Table 5.

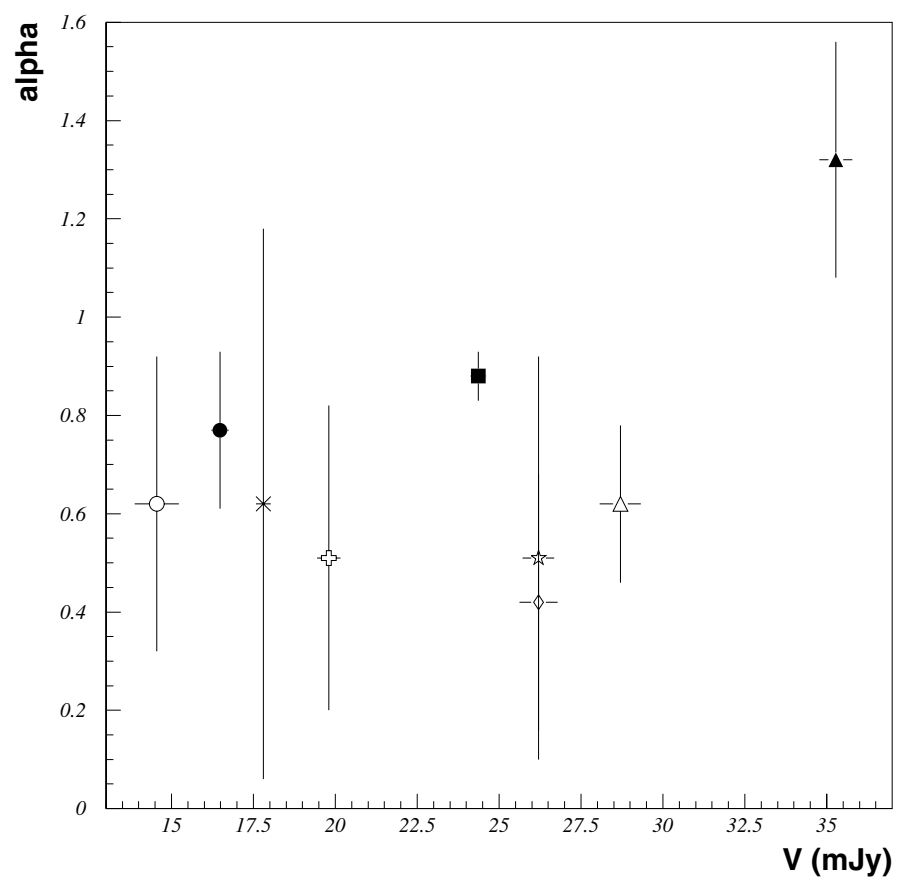

Fig. 12. $\alpha$ vs. $V$ plot for data reported in Fig. 11. Symbols are the same as used in Fig. 11.

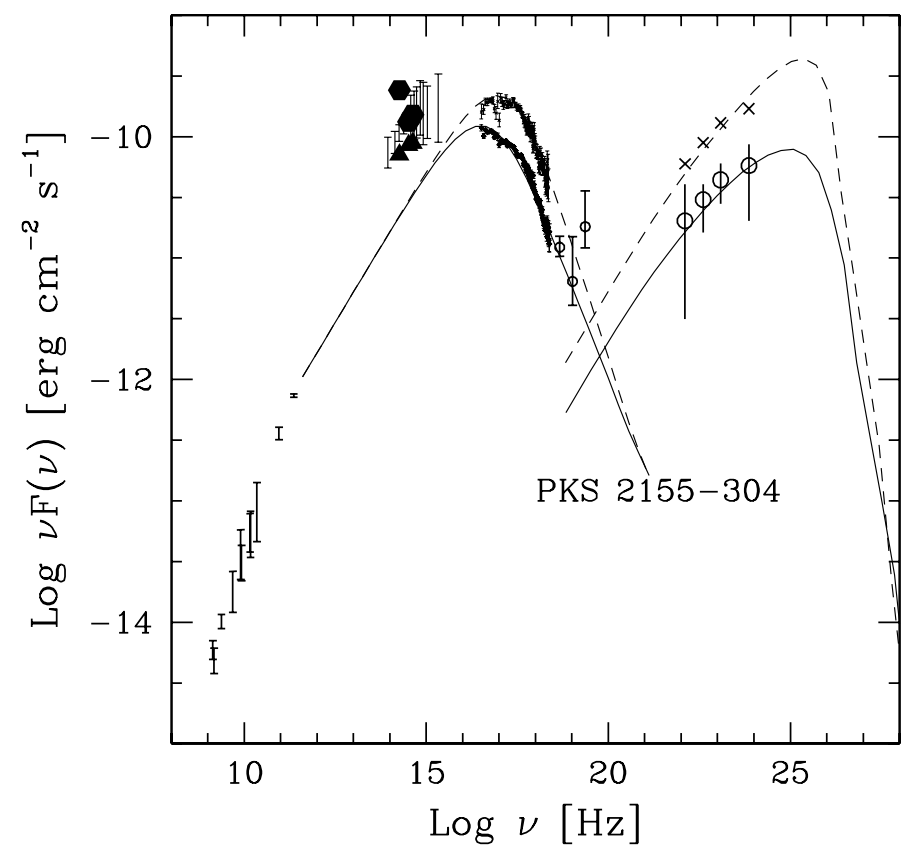

Fig. 13. SED of PKS 2155-304 in two states, adapted from Chiappetti et al. (1999) (see the paper for details). Data from this work are also plotted. Filled triangles correspond to epoch 1 (13/5/2005 data), while filled hexagons belong to epoch 3 data (20/11/2005). Optical, UV and REM data are dereddened using $E(B-V)=0.026$ and parameters given by Cardelli et al. (1989). 